\title{
Experimental Tracheal Allotransplantation Using Omentopexy: Histological Process of Rejection Reaction without Immunosuppression
}

\author{
Shigeharu Moriyama, Nobuyoshi Shimizu and Shigeru \\ Teramoto \\ The Second Department of Surgery, Okayama University \\ Medical School, Okayama 700
}

\begin{abstract}
Moriyama, S., Shimizu, N. and Teramoto, S. Experimental Tracheal Allotransplantation Using Omentopexy: Histological Process of Rejection Reaction without Immunosuppression. Tohoku J. Exp. Med., 1992, 167 (3), 207-218 — A large defect in the thoracic trachea was produced by resecting 10 rings of tracheal cartilage in each of 11 adult mongrel dogs. An allograft of the five tracheal rings was interposed in this site via end-to-end anastomosis and then wrapped with the pedicled omental flap. These animals received no immunosuppressive therapy. The pseudostratified tracheal epithelia were remained and the graft revascularization through the omental pedicle proved to be established within about one week after surgery without immunosuppression. Marked mononuclear cell infiltration was observed beneath the epithelia and around the capillaries in the subepithelial layer over one week after surgery, which was followed by phagocytosis of the tracheal cartilages by macrophages. After the completion of rejection reaction, inflammation finally induced scarring or necrosis of the tracheal allograft, resulting in asphyxia or perforation. Only one animal survived 71 days after surgery without graft trouble. However, even in this animal, weak rejection reaction was observed microscopically and the structure of the graft was not that of the normal trachea. We think these results suggest that allotransplantation of trachea induces rejection, and therefore, that the use of immunosuppressants is necessary. trachea ; allotransplantation ; mongrel dog; omentopexy ; rejection
\end{abstract}

Various tracheal prostheses using artificial and autogenous materials have been utilized in tracheal reconstruction following extensive tracheal resection. However, none are physically and physiologically superior to the trachea itself. For many years, numerous trials on tracheal transplantation have not been successful because of the difficulty of revascularization in the tracheal graft. We solved the problem of revascularization by wrapping the graft in an omental pedicle and achieved long-term graft survival using an immunosuppressant

Received January 7, 1992; revision accepted for publication July 22, 1992.

Address for reprints : Shigeharu Moriyama, M.D., the Second Department of Surgery, Okayama University Medical School, 2-5-1, Shikata-Cho, Okayama 700, Japan. 
FK-506 alone (Moriyama et al. 1989). In this paper, we report the histological features of rejection reaction when no immunosuppressant is used in our experimental tracheal allotransplantation using omentopexy in mongrel dogs.

\section{Materials ANd Methods}

Eleven adult mongrel dogs, weighing 8 to $20 \mathrm{~kg}$, were anesthetized with intramuscular ketamine, intravenous sodium pentobarbital $(30 \mathrm{mg} / \mathrm{kg})$, and pancronium bromide $(2 \mathrm{mg})$. The animals were orally intubated and connected to a volume-limited respirator. The animals were placed in a right thoracotomy and midline laparotomy position. The omentum was mobilized through an upper abdominal midline incision, taking care to preserve the vascular supply from the right gastroepiploic artery. The thoracic trachea was dissected and ten tracheal rings were excised through the right fourth intercostal incision. A 5-rings tracheal allograft was interposed in this defect and sutured in place with 4-0 Prolene over-and-over running sutures. The omental pedicle was pulled up into the thoracic cavity through a $3 \mathrm{~cm}$ opening at the right dome of the diaphragm and was wrapped around the whole graft (Fig. 1). After surgery, no immunosuppressive therapy was applied.

Five of the 11 animals were sacrificed several days after operation for histological study of the early phase of rejection reaction. Animals that died were subjected to laparotomy and thoracotomy to determine the cause of death; tracheal graft, including the wrapped omental pedicle, was excised. The three animals (Nos. 3, 7 and 11) were canulated into the right gastroepiploic artery and injected with $20 \mathrm{ml}$ of India ink under $100 \mathrm{~cm}$ of water

A

B

C

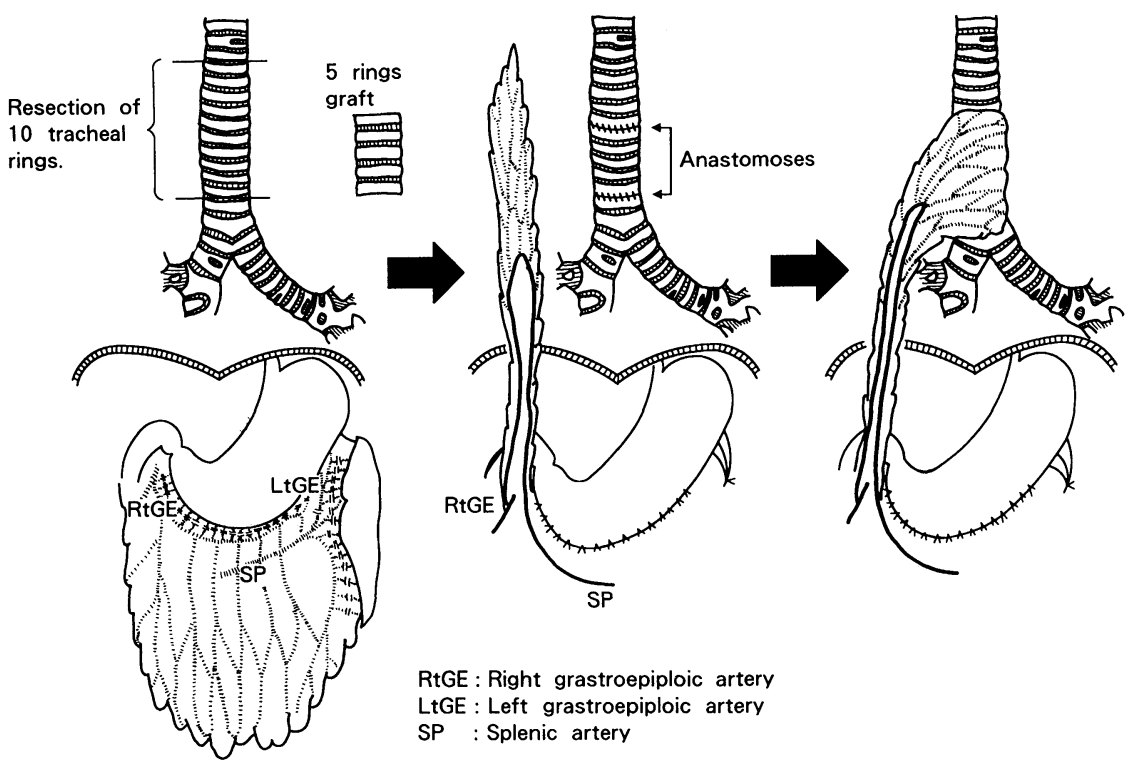

Fig. 1. Schema of experimental procedures of tracheal allotransplantation using omentopexy. (A) Preparation of omental pedicle flap and resection of 10rings thoracic trachea. (B) Transplantation of 5-rings allograft to the tracheal defect, and elevation of the omental pedicle through the right dome of the diaphragma. (C) Wrapping of the omental pedicle around the allograft including both the anastomoses. 
pressure before they were sacrificed to examine revascularization from the omental pedicle to the graft. After macroscopic observation, tracheal grafts were fixed in $10 \%$ formalin solution for histological examination.

All the animals received humane animal care in compliance with the "Principles of Laboratory Care" formulated by the National Society for Medical Research and the "Guide for the Care and Use of Laboratory Animals" prepared by the National Academy of Sciences.

\section{RESUlts}

Results of allograft transplantation without immunosuppression are shown in Table 1. Long-term survival was achieved only in one animal (No.6) that was sacrificed 71 days after transplantation, showing atrophic and yellowish colored transplant compared with normal trachea at necropsy (Fig. 2a). Histological examination of this animal revealed abnormalities; the graft had only one epithelial layer, severe fibrosis in the subepithelial tissue and no regeneration of the tracheal secretory gland (Fig. 2b). Although tracheal cartilages were retained, small recesses containing mononuclear cells at the margin of the cartilages were seen (Fig. 2c). In the other animals, tracheal grafts of the animals which died within about one week after surgery showed relatively normal appearance; pseudostratified columnar ciliary epithelia, normal subepithelial tissue containing secretory glands, and the normal shaped cartilages without cell infiltration (Figs. 3a, 3b). Animal No. 3 was sacrificed on day 7 after injection of India ink. This ink was histologically detected in the capillaries of the subepithelial tissue, showing graft revascularization within the 7 th postoperative day without immunosuppression (Fig. 4). However, the grafts taken from the animals that survived more than one week after surgery showed degeneration,

TABLE 1. Results of allograft transplantation without immunosuppression

\begin{tabular}{|c|c|c|c|}
\hline Dog No. & $\begin{array}{c}\text { Survival } \\
\text { time (days) }\end{array}$ & Cause of death & Graft status \\
\hline 1 & 7 & Sacrificed & Survived \\
\hline 2 & 11 & Prostration & Partial necrosis \\
\hline 3 & 7 & Sacrificed $^{\mathrm{a}}$ & Partial necrosis \\
\hline 4 & 8 & Prostration & Degeneration \\
\hline 5 & 5 & Asphyxia & Partial necrosis \\
\hline 6 & 71 & Sacrificed & Survived \\
\hline 7 & 8 & Sacrificed $^{\mathrm{a}}$ & Partial necrosis \\
\hline 8 & 26 & Asphyxia & Shrinkage \\
\hline 9 & 12 & Prostration & Partial necrosis \\
\hline 10 & 6 & Prostration & Degeneration \\
\hline 11 & 14 & Sacrificed $^{\mathrm{a}}$ & Degeneration \\
\hline
\end{tabular}

${ }^{a}$ India ink was injected from the right gastroepiploic artery before sacrifice. 
shrinkage, or necrosis including those changes of the tracheal epithelia and cartilages. In animal No. 7 on day 8, mononuclear cell infiltration was observed immediately beneath the epithelium (Fig. 5a), around the small vessels in the subepithelial space and the tracheal cartilages (Fig. 5b), and around the minor vessels in the omentum. In animal No. 11 on day 14, the allograft seemed to be surviving, and the subepithelial capillaries were stained with India ink macroscopically (Fig. 6a). Microscopic findings of this animal revealed more severe subepithelial damage than those seen in animal No. 7 (Fig. 6b) and disclosed that the tracheal cartilages were infiltrated and scraped by mononuclear cells (Fig. 6c). The animal No. 8 died of asphyxia on day 26 due to severe stenosis of the airway caused by extreme shrinking of the graft (Fig. 7a). Histological examination revealed the defect of epithelia and the necrotized and scraped tracheal cartilages in the scar tissue (Fig. 7b).

\section{Discussion}

We clarified histologic features of rejection in the tracheal allograft with omentopexy ; well preserved tracheal structure and establishment of revascularization through the omental pedicle within about one week after surgery were followed by marked infiltration of mononuclear cells around the small vessels in the omental pedicle, in the subepithelial interstitial space, and around the capillaries in the subepithelial layer, leading to the phagocytosis of tracheal cartilages by macrophages and finally causing fibrotic changes or necrosis of the transplant. These histologic changes finally brought about severe stenosis or perforation of transplants. Although only one animal (No. 6) lived for 71 days, the histological structure of the allograft was abnormal. This animal alone survived for such a long time, even if a weak rejection reaction was seen, likely because the graft was relatively histocompatible with the recipient.

Ferguson et al. (1950) and Gibson (1967) reported that the trachea has no antigenicity and that its transplantation does not lead to rejection. Since then, various allo- and hetero-transplantation trials have been conducted on the trachea; however, most of them failed, having no immunosuppression and no measures to revascularize the graft.

Some recent reports admit that the trachea is antigeneic and induces rejection. Toivio et al. (1973) performed allotransplantation of the trachea in adult mongrel dogs and observed lymphocyte aggregation at the site of collapse of tracheal cartilage and perivascular lymphocyte cuffing generally distributed in the subepithelial space. Lane et al. (1977) transplanted allogeneic tracheas to the abdominal walls of rats and confirmed the resulting rejection reaction; normal epithelium of the graft was maintained for several days, mononuclear cells consisting principally of small lymphocytes appeared in the epithelium or perivascular sites in the subepithelial layer 10 to 15 days after surgery, and subsequently, activated lymphocytes and lymphoblasts induced tissue injuries. Beigel and 
Müller-Ruchholtz (1984a, b) reported, after a series of experiments, that the more frequent the presensitization of skin grafting is and the higher the histoincompatibility is, the lower the graft survival rate. Beigel et al. (1984) also succeeded in producing an antibody in LEW rats against tracheal epithelium in CAP rats. Bujia et al. (1990) demonstrated HLA class II subregion gene products on human tracheal epithelium and mixed glands. They concluded that tracheal mucosa may be the major antigeneic structure of the trachea and therefore responsible for the immunogeneic action of allogeneic tracheal transplants.

Rose et al. (1979) reported the first clinical case of tracheal allotransplantation without immunosuppression. They did HLA typing and found one HLA-A and two HLA-B mismatches. They obtained 9 weeks graft survival and concluded that tracheal transplants induce only a weak rejection reaction even in the major histoincompatibility. In our experiment, the allograft survived 71 days in one of the 11 cases without immunosuppression. However, even in this case weak rejection reaction was histologically observed. So, we consider that there is a possibility of progressive rejection reaction and long-term follow-up is necessary in the case of Rose et al.

In the present experiment, we obtained results similar to Toivio's and Lane's. Exactly, perivascular cuffing and mononuclear cell infiltration were observed in the immediately beneath the epithelia in the animals on day 8 , in which no epithelial injury was recognized. Severe infiltration of mononuclear cells to the subepithelial tissue, epithelial injuries, cellular infiltration to the tracheal cartilages and melting of the cartilaginous matrix were detected in animals survived more than two weeks. Previously, we reported a good results and long-term graft survival in tracheal allotransplantation using omentopexy with immunosuppressant (Moriyama et al. 1989). We performed the same procedures as present experinent in 28 adult mongrel dogs, except for the administration of immunosuppressant FK-506. Only 2 animals died of rejection or graft necrosis and twenty allografts were proved to be survived; the viability of the residual 6 grafts was not determined, because the animals died of other diseases within 5 days. Eighteen animals survived for more than two weeks and no histological sign of rejection, as mentioned above, was not seen in their allografts. These results suggest that tracheal allograft induces rejection reaction if no immunosuppressant were administered, and therefore, that the use of immunosuppressants is necessary in tracheal allotransplantation.

\section{References}

1) Beigel, A. \& Müller-Ruchholtz, W. (1984a) Tracheal transplantation. I. The immunogenic effect of rat tracheal transplants. Arch. Otorhinolaryngol., 240, 185-192.

2) Beigel, A. \& Müller-Ruchholtz, W. (1984b) Tracheal transplantation. II. Influence of genetic difference and degree of sensitization on reactions to the tracheal transplant. Arch. Otorhinolaryngol., 240, 217-225.

3) Beigel, A., Steffens-Knutzen, R., Müller, B., Schumacher, U. \& Stein, H. (1984) 
Tracheal transplantation. III. Demonstration of transplantation antigens on the tracheal mucosa of inbred rat strains. Arch. Otorhinolaryngol., 241, 1-8.

4) Bujia, J., Wilmes, E., Hammer, C. \& Kastenbauer, E. (1990) Tracheal transplantation: Demonstration of HLA class II subregion gene products on human trachea. Acta Otolaryngol., 110, 179-154.

5) Ferguson, D.J., Wild, J.J. \& Wangensteen, O.H. (1950) Experimental resection of the thoracic trachea. Surgery, 28, 597-619.

6) Gibson, T. (1967) The transplantation of cartilage. The College of Pathologists. Tissue and Organ Transplantation. B.M.A. House, Tavistock Square, London W.C. 1, England.

7) Lane, B.P., Habicht, G.S. \& Jasper, G.S. (1977) Lymphocyte-epithelium interaction during rejection of nonisogenic rat tracheal grafts. Am. J. Pathol., 86, 71-77.

8) Moriyama, S., Shimizu, N. \& Teramoto, S. (1989) Experimental tracheal allotransplantation using omentopexy. Transplant. Proc., 21, 2596-2600.

9) Rose, K.G., Sesterhenn, K. \& Wustrow, F. (1979) Tracheal allotransplantation in man. Lancet, 1, 433.

10) Toivio, I., Siirala, U., Lauerma, S., Rapo, S., Tallberg, T., Mahlberg, K., Tapaninen, J. \& Meurala, H. (1973) Homotransplantation of canine trachea after denervation of the spleen. Acta Otolaryng., 75, 127-131. 

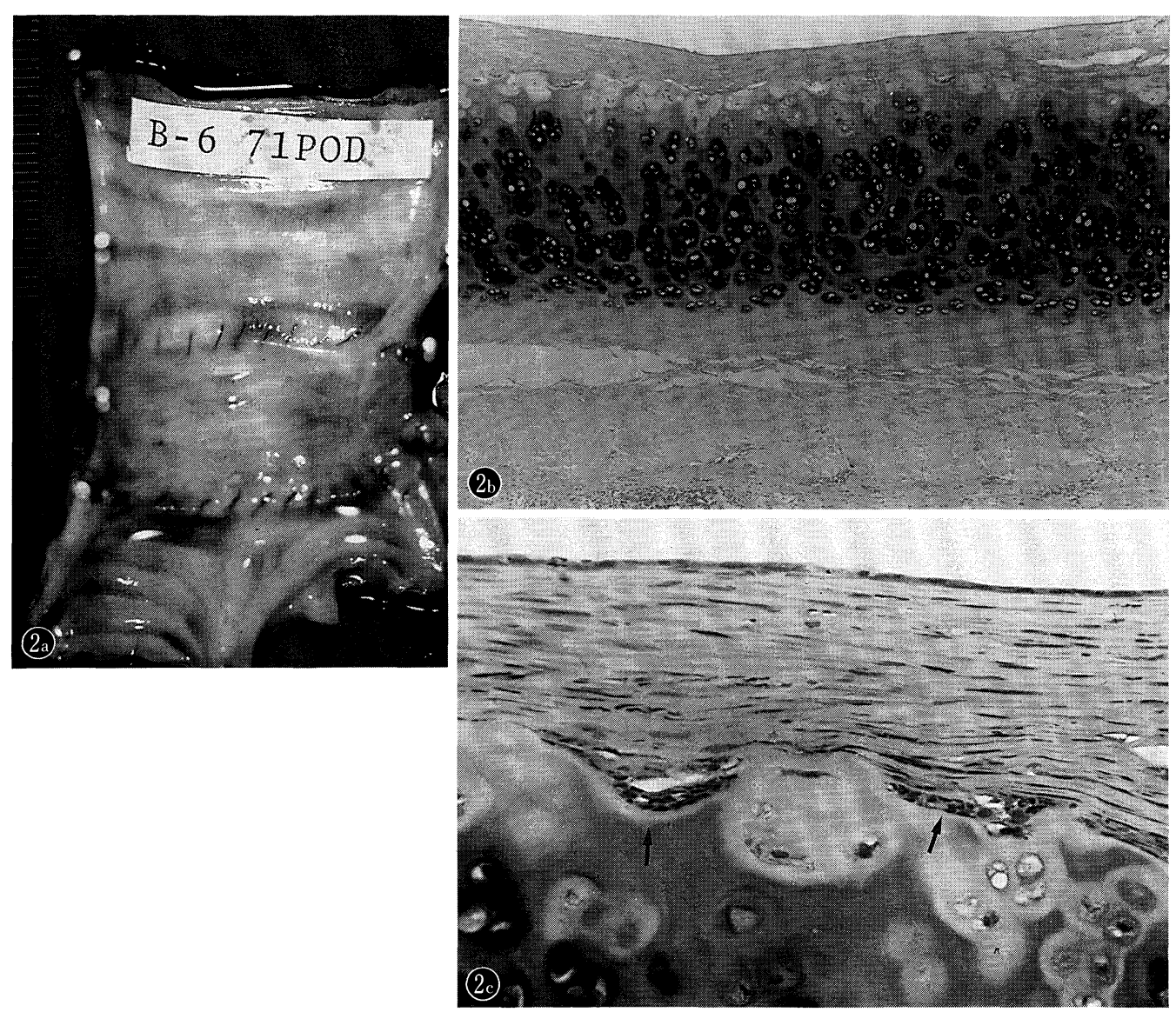

Fig. 2. Macroscopic and microscopic findings of the allograft of dog No. 6 on postoperative day (POD) 71.

(a) Macroscopic findings. The allograft is slightly shrunken and the epithelia is not translucent. Either of the anastomoses is not shrunken and the airway is not narrowed.

(b) Microscopic findings (hematoxylin and eosin stain $[\mathrm{H} \& \mathrm{E}], \times 16$ ). The tracheal epithelium is only one layer. Subepithelial layer is composed of fibrous tissue, and tracheal secretory gland is not seen, which is abnormal compared with normal tracheal structure. The tracheal cartilage apparently maintains its volume; however, small recesses are seen on the surface of the cartilage.

(c) Microscopic findings $(\mathrm{H} \& \mathrm{E}, \times 50)$. The small recesses on the surface of the cartilage contain mononuclear cells including macrophages (arrows). 

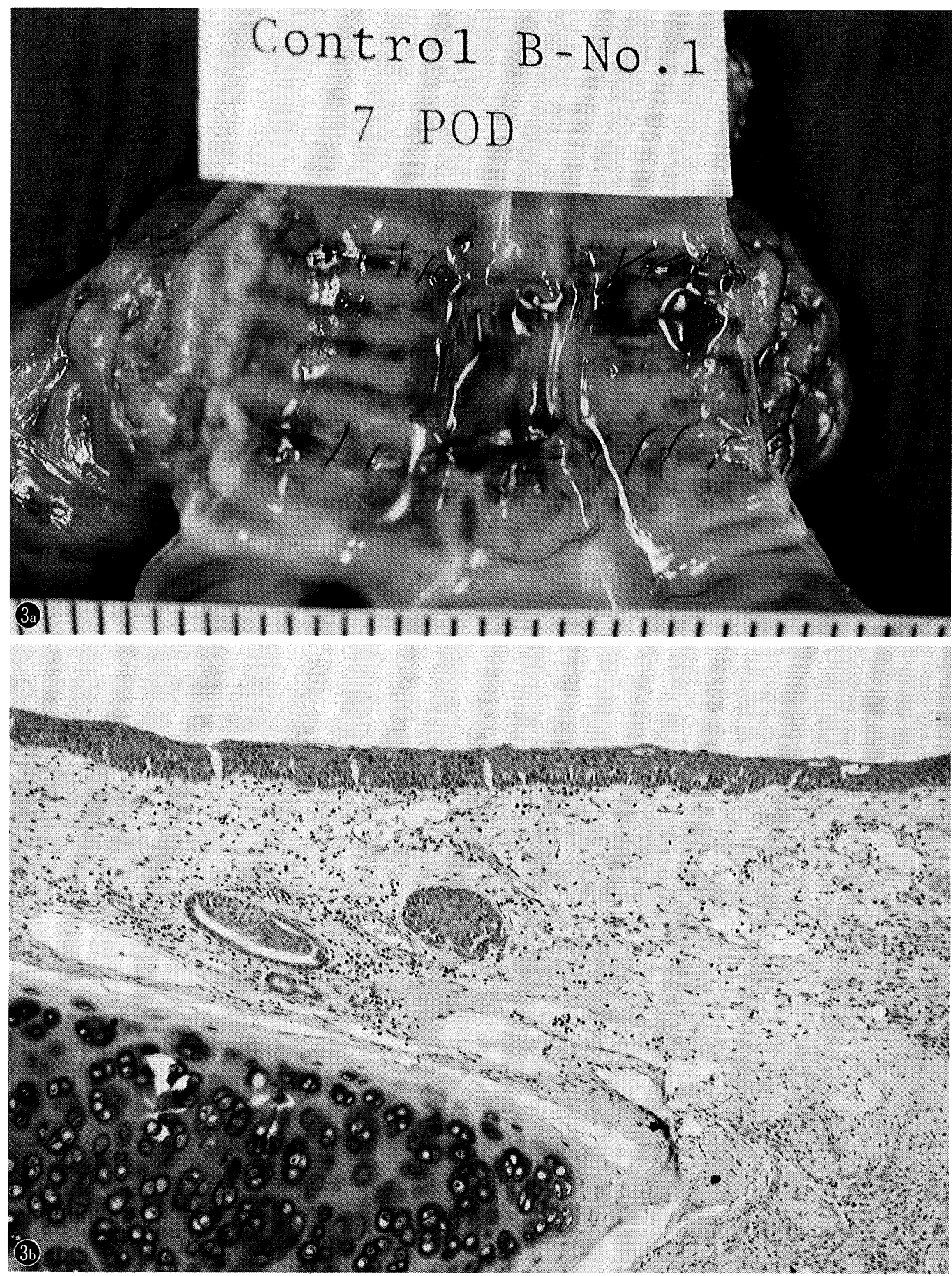


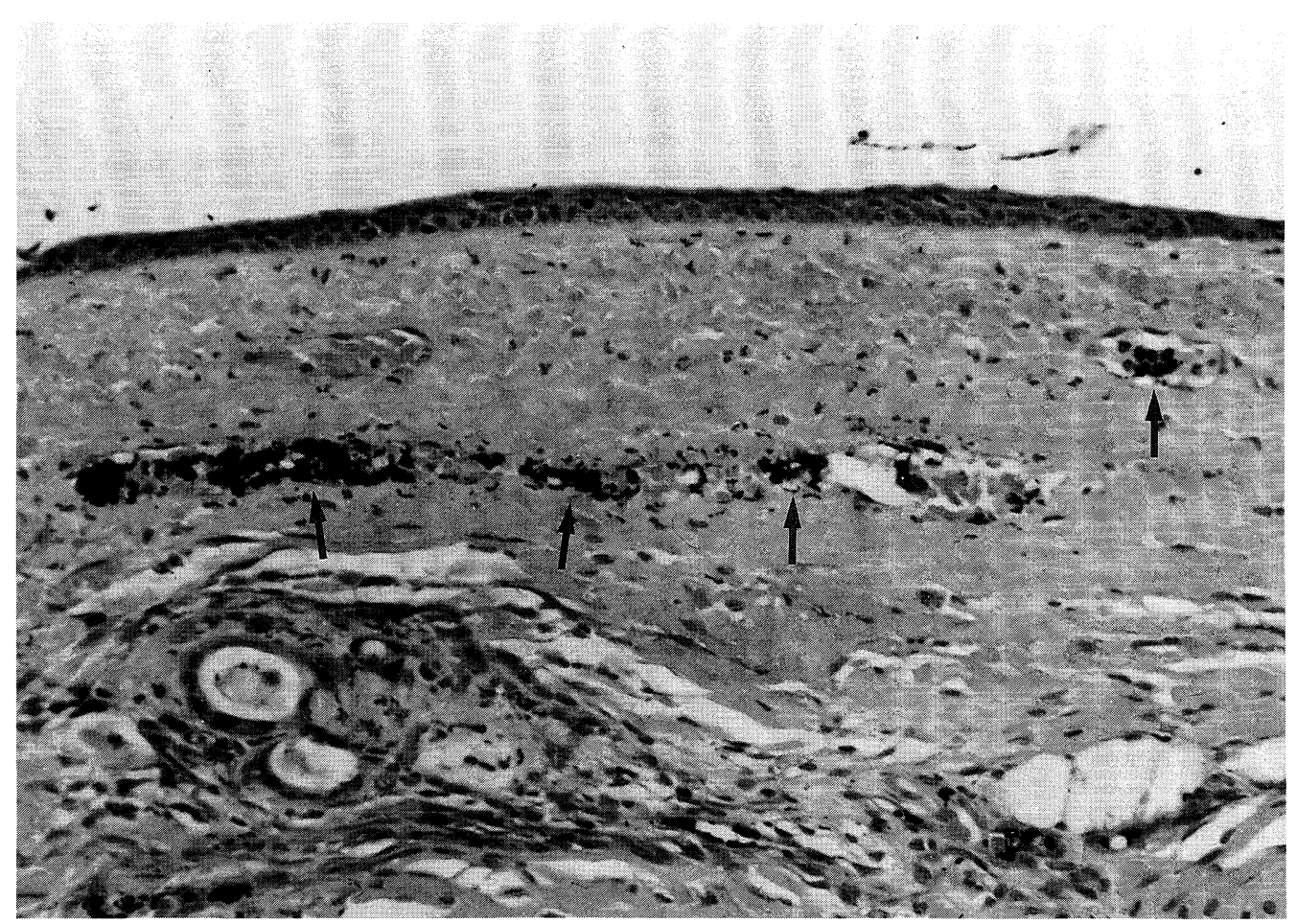

Fig. 4. Microscopic findings of the allograft of dog No. 3 on POD $7(\mathrm{H} \& \mathrm{E}, \times 5)$. Although degeneration of the subepithelial tissue of the allograft is severe in this case, India ink injected from the right gastroepiploic artery before sacrifice is seen in the capillaries of the subepithelial layer (arrows).

Fig. 3. Macroscopic and microscopic findings of the allograft of on POD 7.

(a) Macroscopic findings. The specimen is longitudinally cut in the midline of the tracheal cartilage. The membranous portion is seen in the center of the specimen. The tracheal epithelium is translucent and the tracheal cartilages are seen.

(b) Microscopic findings $(\mathrm{H} \& \mathrm{E}, \times 8)$. The pseudostratified columnar epithelia, tracheal secretory glands, subepithelial tissue, and the tracheal cartilage remain normal. No recess is seen on the surface of the cartilage. 

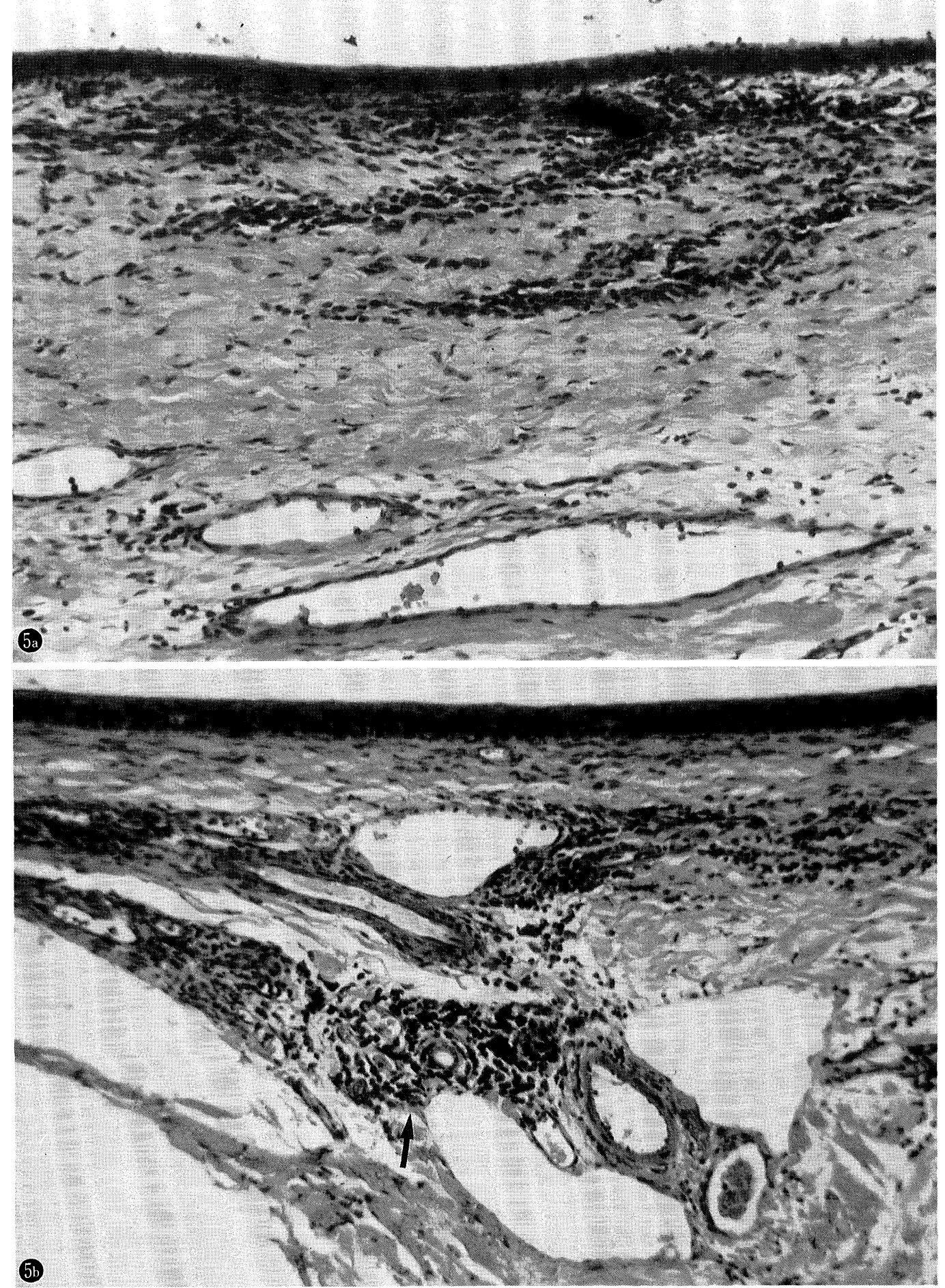

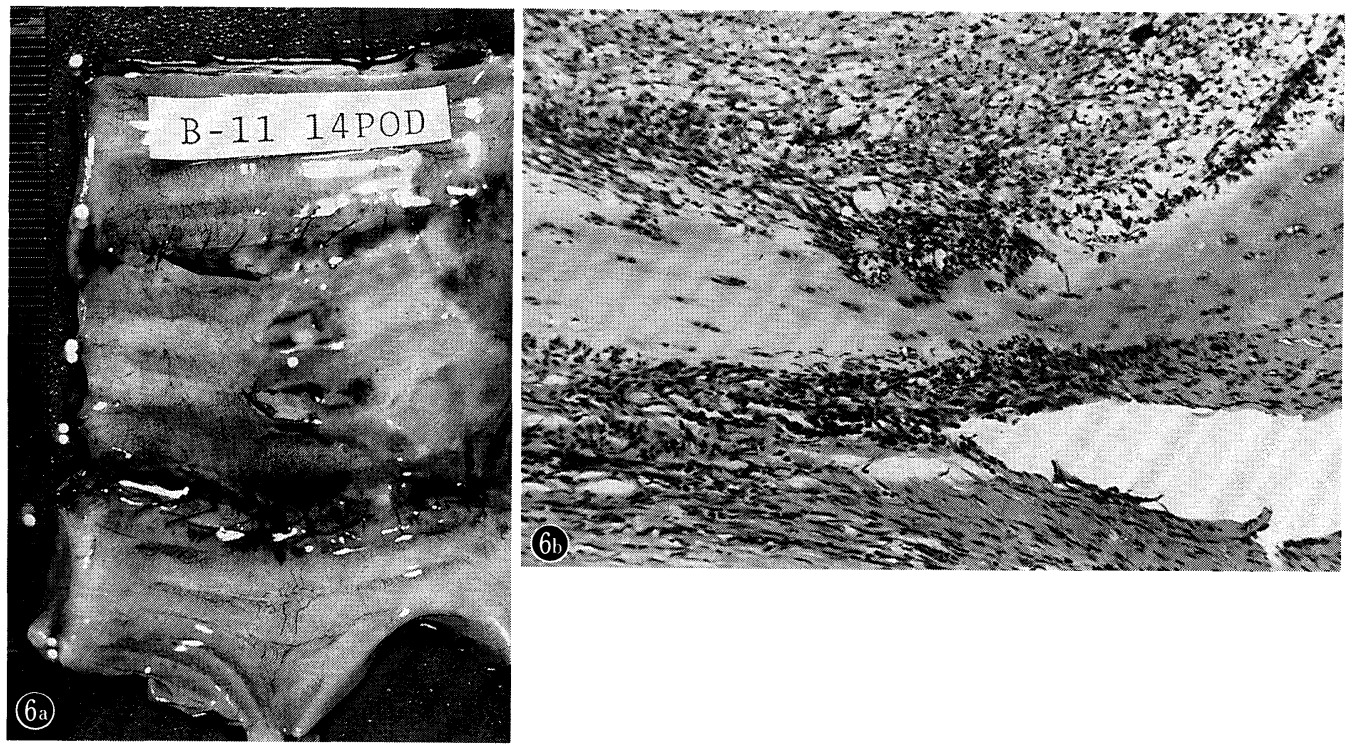

Fig. 6. Macroscopic and microscopic findings of the allograft of dog No. 11 on POD 14.

(a) Macroscopic findings. India ink was injected into the animal before sacrifice. The ink is seen in the subepithelial capillaries of both the allograft and the recipient trachea. Degeneration is seen on the right half of this specimen.

(b) Microscopic findings $(\mathrm{H} \& \mathrm{E}, \times 50)$. Massive mononuclear cell infiltration into the cartilage is seen. The cartilaginous matrix is greatly scraped.

Fig. 5. Microscopic findings of the allograft of dog No. 7 on POD 8. (H \& E, $\times 50$ ). (a) Tracheal epithelia remained in some layers. Mononuclear cells infiltrate beneath the epithelia.

(b) Mononuclear cell infiltration is also seen around the deep subepithelial small vessels ("perivascular cuffing") (arrow). 

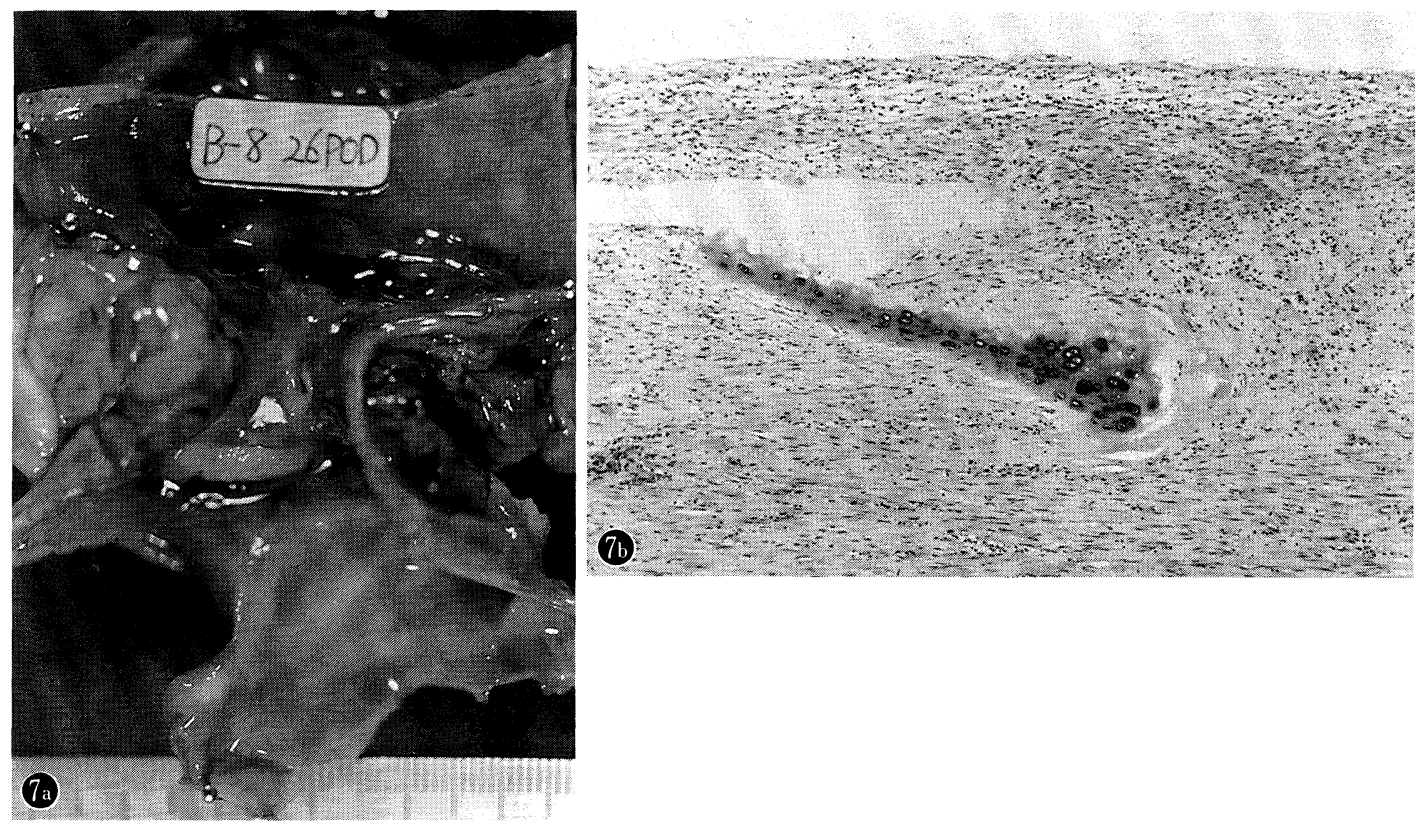

Fig. 7. Macroscopic and microscopic findings of the allograft of dog No. 8 on POD 26.

(a) Macroscopic findings. The allograft is remarkably shrunken and the airway is narrowed. Both the anastomoses are clear and no granulation is seen.

(b) Microscopic findings $(\mathrm{H} \& \mathrm{E}, \times 50)$. The epithelium is defective and the cartilage is almost completely melt in the granulation tissue. 